\title{
Schwermetalle im Bodensee
}

\section{Mechanismen der Konzentrationsregulierung}

\section{Sigg, M. Sturm und W. Stumm}

Eidgenössische Anstalt für Wasserversorgung, Abwasserreinigung

und Gewässerschutz (EAWAG) der ETH Zürich, CH-8600 Dübendorf

\section{Mart und H.W. Nürnberg}

Institut für Angewandte Physikalische Chemie, Chemie Department,

Kernforschungsanlage Jülich, D-5170 Jülich

Aus der Zunahme der Schwermetallkonzentration in den obersten (jüngsten) Schichten der Sedimente des Bodensees und anderen Seen ließ 3 sich die Zunahme der Belastung mit Schwermetallen, insbes. mit atmophilen Elementen, $\mathrm{Cu}, \mathrm{Cd}, \mathrm{Zn}$, $\mathrm{Pb}$, im Laufe der letzen Jahrzehnte ableiten [1]. Obschon die Metallbelastung europäischer Seen durch Zuflüsse und Deposition aus der Atmosphäre um ein bis zwei Größenordnungen größer ist als diejenige der Ozeane, weisen Seen und Meere ähnlich niedrige Konzentrationen an gelösten Metallen auf (Tabelle 1). Offensichtlich sind in den Seen äußerst effiziente Eliminationsmechanismen wirksam. Sedimentierende biogene Partikel (Phytoplankton und biologisches Debris) sowie eingebrachte Mineralteilchen sind Träger für den Transport der Metalle in die Sedimente.

Um Einblick in die Mechanismen der Regulierung der Metallkonzentrationen im Bodensee zu gewinnen, wurden während der Sommerstagnationsperiode in ca. 1monatlichen Abständen Tiefenprofile der Konzentrationen von im Wasser gelöstem und in Schwebestoffen enthaltenem $\mathrm{Fe}$, $\mathrm{Mn}, \mathrm{Cd}, \mathrm{Cu}, \mathrm{Zn}, \mathrm{Pb}$ und anderer limnologischer Parameter sowie die Sedimentationsrate und die Zusammensetzung des sedimentierenden Materials in der Mitte des Sees zwischen Huttwil und Fischbach (Tiefe des Sees ca. $250 \mathrm{~m}$ ) bestimmt. Sedimentierende Partikel wurden in Sedimentpfannen (50 und $240 \mathrm{~m}$ Tiefe) aufgefangen und analysiert. Die Methoden der Probennahme und der Analyse unter Einsatz von Voltammetrie und AAS wurden bereits beschrieben [4-7].

Tabelle 1 vergleicht Belastung, Konzentration von Metallen und Sedimentationsraten im Nordatlantik mit jenen des Bodensees. Die gegenüber dem Atlantik viel hö-
Tabelle 1. Belastung und Elimination der Schwermetalle im Bodensee im Vergleich zum Nordatlantik

\begin{tabular}{|c|c|c|c|c|c|}
\hline & & $\mathrm{Cu}$ & $\mathrm{Pb}$ & $\mathrm{Cd}$ & $\mathrm{Zn}$ \\
\hline \multicolumn{6}{|l|}{ Bodensee } \\
\hline Atmosphärischer Eintrag ${ }^{a}$ & [ng cm $\left.{ }^{-2} \mathrm{Jahr}^{-1}\right]$ & 714 & 11000 & 20 & 8400 \\
\hline $\begin{array}{l}\text { Fracht der sedimentierenden } \\
\text { Partikel }^{\text {b }}\end{array}$ & {$\left[\mathrm{ng} \mathrm{cm}^{-2} \mathrm{Jahr}^{-1}\right]$} & 6500 & 9500 & $(100)^{\mathrm{c}}$ & 36000 \\
\hline $\begin{array}{l}\text { Konzentration im See } \\
\text { Nordatlantik }\end{array}$ & [ng/l] & $300-800$ & $50-100$ & $6-20$ & $1000-4000$ \\
\hline $\begin{array}{l}\text { Atmosphärischer Eintrag } \\
\text { (Partikel) (aus [2]) }\end{array}$ & [ng cm ${ }^{-2} \mathrm{Jahr}^{-1}$ ] & 25 & 310 & - & 130 \\
\hline $\begin{array}{l}\text { Fracht der sedimentierenden } \\
\text { Partikel }\end{array}$ & [ng cm-2 $\left.\mathrm{Jahr}^{-1}\right]$ & 234 & 330 & - & 1040 \\
\hline $\begin{array}{l}\text { Konzentration im Meer } \\
\quad(\text { aus }[3,5,8])\end{array}$ & {$[\mathrm{ng} / \mathrm{l}]$} & $\begin{array}{l}100 \\
(30-300)\end{array}$ & $\begin{array}{c}3 \\
(1-15)\end{array}$ & $\begin{array}{l}10 \\
(1-120)\end{array}$ & $\begin{array}{l}100 \\
(10-600)\end{array}$ \\
\hline
\end{tabular}

a Abgeschätzt aus Messungen der EAWAG für die nördliche Schweiz, Schweiz. Jahrbuch für Hydrologie 1976-1979

b Unsere Messungen; "Berechnet aufgrund der Zusammensetzung der Seesedimente here Sedimentationsrate im Bodensee, hervorgerufen vor allem durch die größere Produktion (Photosynthese) biogener Partikel, ist primär für die wirkungsvolle Metall-Elimination verantwortlich. Dementsprechend wurde auch der größte Teil der von uns untersuchten in den See eingebrachten Elemente durch Sedimentation aus dem See entfernt. Während der meisten Zeit der Meßperiode machte der biogene Anteil mehr als die Hälfte der sedimentierenden Partikel (Trockengewicht) aus; nur im Oktober war der allochthone Anteil größer als der autochthone.

Die Zusammensetzung des in 50 und $240 \mathrm{~m}$ aufgefangenen sedimentierenden
Materials (Tabelle 2) weist in den verschiedenen Proben für viele der aufgeführten Elemente eine erstaunlich konstante $\mathrm{Zu}$ sammensetzung auf. In Bezug auf die Nährstoffelemente entspricht die Zusammensetzung weitgehend den im See gefundenen stöchiometrischen Konzentrationsveränderungen. In erster Näherung kann man postulieren, daß bei der Produktion der biogenen Partikel diese Elemente in der der Partikelzusammensetzung entsprechenden Proportion aus den oberen Wasserschichten entzogen und bei der teilweisen respiratorischen Auflösung in die werden: unteren Wasserschichten zurückgegeben

$$
\begin{aligned}
& 100 \mathrm{Ca}^{2+}+200 \mathrm{HCO}_{3}{ }^{-}+12 \mathrm{NO}_{3}{ }^{-}+14 \mathrm{SiO}_{2}+\mathrm{HPO}_{4}{ }^{2-}+14 \mathrm{H}^{+}+0,04 \mathrm{Zn}^{2+}+0,01 \mathrm{Cu}^{2+} \\
& \overbrace{\text { Respiration }}+0,0001 \mathrm{Cd}^{2+}+0,004 \mathrm{~Pb}^{2+} \\
& \begin{array}{l|l}
\text { Photosynthese } & \text { Respiration }
\end{array} \\
& \left\{\left(\mathrm{CaCO}_{3}\right)_{100}\left(\mathrm{SiO}_{2}\right)_{14}\left(\mathrm{CH}_{2} \mathrm{O}\right)_{100}\left(\mathrm{NH}_{3}\right)_{12}\left(\mathrm{H}_{3} \mathrm{PO}_{4}\right) \mathrm{Zn}_{0,04} \mathrm{Cu}_{0,01} \mathrm{Cd}_{0.0001} \mathrm{~Pb}_{0.004}\right\}+124 \mathrm{O}_{2} \\
& \text {,biogene Partikel““ }
\end{aligned}
$$


Tabelle 2. Zusammensetzung der sedimentierenden Teilchen im Bodensee

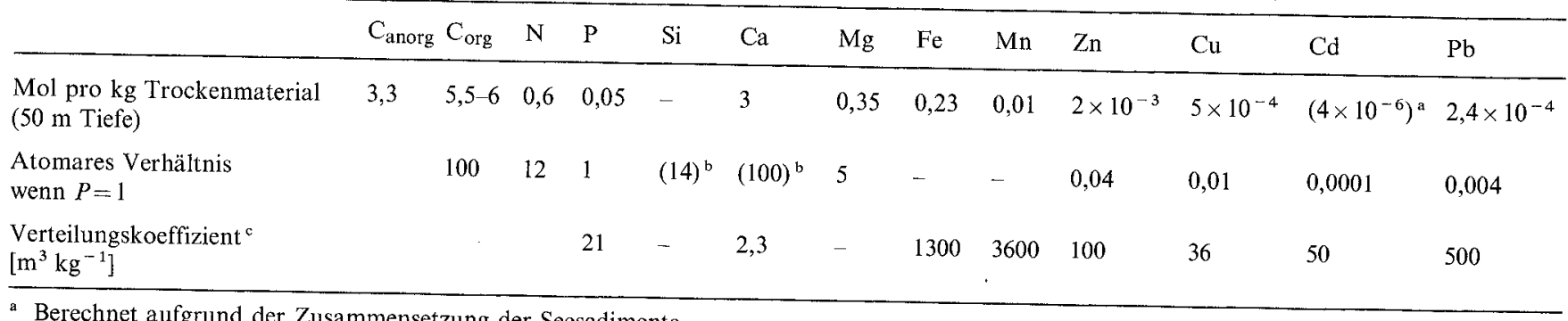

Berechnet aufgrund der Zusammensetzung der Seesedimente

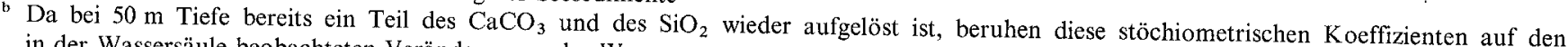
in der Wassersäule beobachteten Veränderungen der Wasserzusammensetzung

c Konzentration feste Phase $\left[\mathrm{g} \mathrm{kg}^{-1}\right]$ :Konzentration in Lösung $\left[\mathrm{g} \mathrm{m}^{-3}\right]$

Stationärzustands-Modellberechnungen, in denen die Frachten der Metalleinträge (über Atmosphäre und Flüsse) den Frachten des Metallabtransportes (Sedimentation und Ausflüsse) gleichgesetzt werden, ergeben für $\mathrm{Zn}, \mathrm{Cd}, \mathrm{Cu}$ und $\mathrm{Pb}$ Konzentrationen von $1,5,0,005,1$ und $0,1 \mu \mathrm{g} / \mathrm{l}$; diese entsprechen, innerhalb eines Faktors 2, den gemessenen Konzentrationen. Das bestätigt die Bedeutung der Sedimentation als Eliminationsmechanismus.

Representative Konzentrationsprofile für diese Elemente sind in Fig. 1 wiedergegeben. Die Profile sind qualitativ in Übereinstimmung mit der Vorstellung, daß das wachsende Phytoplankton und allochthone Partikel in den Oberflächenschichten des Sees dem Wasser Schwermetalle, zusammen mit Nährstoffen, entziehen. Die Zersetzung des biologischen Debris unterhalb der photischen Zone führt zu einer teilweisen Freisetzung der Metalle. Daß die Korrelation zwischen Metall- und Nährstoffkonzentration nicht so ausgeprägt ist wie in den Meeren [8], ist darauf zurückzuführen, daß der Metalleintrag an der Wasseroberfläche, insbesondere aus der Atmosphäre, recht groß ist. Der Einfluß des atmosphärischen Eintrags ist für $\mathrm{Pb}$ besonders deutlich. Ferner besteht in den sedimentnahen Wasserschichten ein zusätzliches partikuläres Förderband aus Eisenund Manganoxiden; diese entstehen vor allem im späteren Teil der Stagnationsperiode durch die Oxidation des aus teilweise

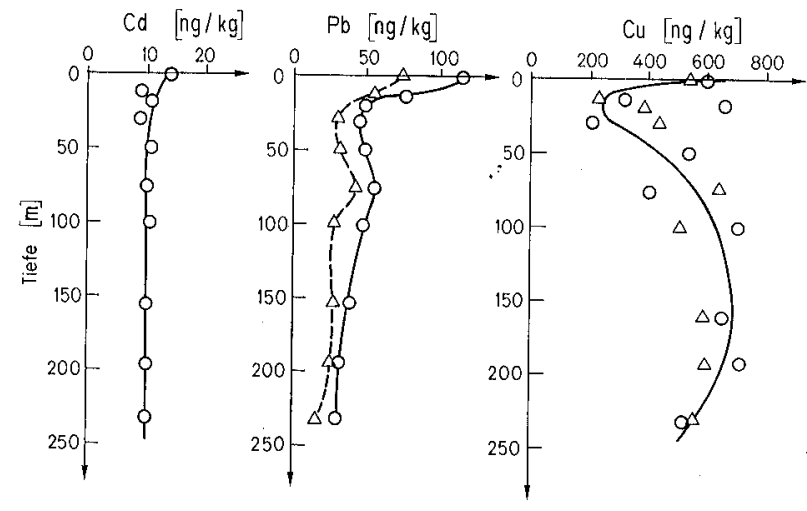

Fig. 1. Konzentrationsprofile von $\mathrm{Cd}(\mathrm{II}), \mathrm{Pb}(\mathrm{II})$ und $\mathrm{Cu}(\mathrm{II})$, $O$ insgesamt, $\triangle$ filtriert

anoxischen Sedimenten rückdiffundierenden $\mathrm{Fe}(\mathrm{II})$ und $\mathrm{Mn}(\mathrm{II})$.

Wir sind Kollegen aus verschiedenen Instituten für ihre Unterstützung dankbar, insbes. Dr. Stabel und Prof. Tilzer (Limnologisches Institut der Universität Konstanz), Dr. J. Müller, Dr. U. Zeh (Universität München), Dr. Zahner, Dr. Rossknecht, Dr. Einzele (Institut für Seenforschung und Fischereiwesen Langenargen), Dr. Valenta, P. Klahre, H. Rützel (Institut für angewandte phys. Chemie der Kernforschungsanlage Jülich), Dr. Davis (EAWAG). Die Arbeiten wurden teilweise vom Schweizerischen Nationalfonds unterstützt.

Eingegangen am 5. Juli 1982
1. Müller, G.: Naturwissenschaften 64,427 (1977); Imboden, D.M., Tschopp, J., Stumm, W.: Schweiz. Z. Hydrol. 42, 1 (1980)

2. Buat-Menard, P., Chesselet, R.: Earth Planet. Sci. Lett. 42, 399 (1979)

3. Schaule, B., in: Lead in Marine Environment (M. Branica, Z. Konrad, eds.). Oxford: Pergamon 1980; Mart, L., et al. : Sci. Tot. Environm. (im Druck)

4. Mart, L.: Fresenius Z. Anal. Chem. 299, 97 (1979)

5. Mart, L., Nürnberg, H.W., Dyrssen, D., in : Trace Metals in Sea Water (C.S. Wong, K. Bruland, eds.). New York: Plenum Press 1982

6. Mart, L., Nürnberg, H.W., Valenta, P. : Fresenius Z. Anal. Chem. 300, 350 (1980)

7. Sigg, L., et al.: Thal. Jugosl. (im Druck)

8. Bruland, K.W.: Earth Planet. Sci. Lett. 47, 176 (1980); Boyle, E.A., Huested, S.S., Jones, S.P.: J. Geophys. Res. 86, 8048 (1981) 